\title{
Dynamic viscous permeability of an open-cell aluminum foam: Computations versus experiments
}

\author{
Camille Perrot, ${ }^{\text {a) }}$ Fabien Chevillotte, and Raymond Panneton \\ Groupe d'Acoustique de l'Université de Sherbrooke (GAUS), Department of Mechanical Engineering, \\ Université de Sherbrooke, Quebec J1 K 2R1, Canada
}

(Received 26 July 2007; accepted 4 November 2007; published online 25 January 2008)

\begin{abstract}
Is it possible to find a two-dimensional (2D) periodic unit cell representative of the dynamic viscous dissipation properties of a real porous media? This is a challenging question addressed in this paper through a review of tools and methods of experimental and computational micro(poro)mechanics. The combination of advanced experimental imaging and numerical homogenization techniques provides a unique opportunity to understand and assess the limits of two-dimensional models of microstructures, as a potential basis for the engineering prediction of macroscopic properties of acoustical materials. This is illustrated for a real sample of open-cell aluminum foam. The conclusion, based on this analysis, is that the $2 \mathrm{D}$ periodic foam model geometry provides a reliable estimate of the dynamic permeability, except in the low frequency range. This is not surprising because in the $2 \mathrm{D}$ periodic foam model geometry, ligaments are always perpendicular to the flow direction, thus decreasing artificially the static permeability of the viscous flow. (c) 2008 American Institute of Physics. [DOI: 10.1063/1.2829774]
\end{abstract}

\section{INTRODUCTION}

The general objective of this work is the determination of the acoustical macrobehavior at the local scale of real porous media. In this purpose, one needs first to determine the local geometry of the media, and second to solve the partial differential equations governing the propagation phenomena of an acoustic wave such as the frequency dependent thermal and viscous dissipation effects. The first step is made feasible by the modern technique of x-ray computed microtomography $(\mu \mathrm{CT})$. Various geometrical properties can be determined on the experimental sample with a view to characterize the representative parameters of its cellular morphology and reconstruct the porous medium by means of a threedimensional (3D) idealized periodic unit cell (PUC). This technique was applied to the characterization of open-cell aluminum foam samples, and good predictions of the purely geometrical macroscopic properties were obtained. ${ }^{1}$ Simulation results of frequency dependent heat conduction through 3D reconstructed unit cells of an open-cell aluminum foam under acoustic excitations have also been recently reported. The random-walker algorithm was applied to the calculation of the dynamic bulk modulus and compared to laboratory measurements with good agreements. ${ }^{2}$

Several authors have reported analytical, numerical, and experimental studies of effective viscous dissipation properties of periodic porous structures in the long wavelength limit. ${ }^{3-7}$ Oscillatory flows have been studied analytically in straight capillaries and between two parallel plates. ${ }^{3,4} \mathrm{Ad}-$ vanced numerical investigations consist in solving the steady Stokes, unsteady Stokes, and electric boundary value problems governing viscous dissipation mechanisms in the low frequency, fully dynamic, and high frequency regimes, re-

\footnotetext{
${ }^{a)}$ Author to whom correspondence should be addressed. Electronic mail: camille.perrot@usherbrooke.ca.
}

spectively. A pioneering work was a finite element approach for the sinusoidally modulated tube, the fused-spherical-bead lattice, and the fused diamond lattice by Sheng and Zhou. ${ }^{5}$ They computed the dynamic viscous permeability $k(\omega)$, as well as other asymptotic parameters such as the static viscous permeability $k_{0}$, the tortuosity $\alpha_{\infty}$, and the surface length parameter $\Lambda$ as defined by Johnson et al. ${ }^{6}$ Charlaix et $a .^{7}{ }^{2}$ reported experimental measurements of the dynamic viscous permeability performed on capillary tubes and porous media made of fused glass beds and crushed glass of various sizes. They obtained good agreements with Sheng and Zhou numerical results. Many theoretical, numerical, and experimental investigations have been performed to predict these quantities in periodic structures. Key works include Refs. 8-14; however, no foam model geometry has been investigated.

The purpose of this paper is to present a twodimensional (2D) PUC, which introduces an underlying foam structure, but only makes use of purely geometrical macroscopic parameters deduced from a reconstructed 3D PUC of a real aluminum foam sample. This allows a direct assessment of the merit of the $2 \mathrm{D}$ model with respect to the dynamic viscous dissipation properties criteria. Special emphasis is put on the limitations of the 2D PUC model and its rendering in the viscous dissipation properties when compared to those of a real foam sample.

This paper is organized as follows. In Sec. II, the basic equations used to calculate the various macroscopic parameters under consideration and the dynamic viscous permeability are presented. In Sec. III, the numerical values of the dynamic viscous permeability are compared with the models of Johnson et al. ${ }^{6}$ and Pride et al. ${ }^{11}$ for the proposed 2D periodic foam model geometry. The predictions are com- 
pared to measurements performed at normal incidence in an impedance tube. Finally, Sec. IV draws the main conclusions of this work.

\section{THEORETICAL BACKGROUND}

\section{A. Dynamic viscous dissipation properties}

The flow of a viscothermal fluid in a motionless homogeneous porous structure can be described as follows. ${ }^{15}$ The angular frequency is $\omega$ and the time dependence is $\exp (-i \omega t)$. The statistical properties of the porous frame can be defined in homogenization volumes with dimensions much smaller than the wavelength of the acoustic waves that propagate in the saturating fluid. The microscopic quantities that describe the flow (pressure $p$ and velocity $\mathbf{v}$ ) present variations at the microscopic scale in the homogenization volume. To smooth out these variations and leave only the macroscopic variations, the symbol \langle\rangle , indicating a fluid phase average, is introduced. By definition, it relates the macroscopic variable $\langle a\rangle$ at $\mathbf{r}$ to the microscopic variable $a$ by

$$
\langle a\rangle(\mathbf{r}, t)=\int_{\Omega_{s}+\Omega_{f}} I(\mathbf{r}+\mathbf{x}) f(\mathbf{x}) a(\mathbf{r}+\mathbf{x}, t) d^{3} \mathbf{x},
$$

where $I$ is the characteristic function of the fluid phase ( 1 in the fluid $\Omega_{f}$ and 0 in the solid $\Omega_{s}$ ), and $f$ is a filtering smooth function nonzero only in some (homogenization) region surrounding $\mathbf{x}=0$ and normalized to unity $\int_{\Omega_{s}+\Omega_{f}} I(\mathbf{r}$ $+\mathbf{x}) f(\mathbf{x}) d^{3} \mathbf{x}=1$.

At a given frequency, two equivalent relations linking the gradient of the macroscopic pressure to the macroscopic velocity are

$$
\rho_{0} \alpha_{i j}(\omega) \frac{\partial\langle\mathbf{v}\rangle_{j}}{\partial t}(\mathbf{r})=-\nabla_{i}\langle p\rangle(\mathbf{r}),
$$

where $\rho_{0}$ is the equilibrium density of the fluid, and

$$
\phi\langle\mathbf{v}\rangle_{i}(\mathbf{r})=-\frac{1}{\eta} k_{i j}(\omega) \nabla_{j}\langle p\rangle(\mathbf{r}),
$$

where $\phi$ is the porosity and $\eta$ is the viscosity of the fluid. The symbol $\nabla$ corresponds to $\partial / \partial \mathbf{r}$ before \langle\rangle and to $\partial / \partial \mathbf{x}$ inside \langle\rangle . The vector $\mathbf{n}$ is the unit outward normal from the fluid domain. Two second order tensors, the dynamic tortuosity $\alpha_{i j}(\omega)$ and the dynamic permeability $k_{i j}(\omega)$, are defined by Eqs. (2) and (3). They only depend on the frequency and on the geometry of the porous structure. These two quantities, previously defined and studied by Johnson et al. ${ }^{6}$ characterize the response of the fluid to a spatially constant oscillating pressure gradient. When frequency decreases, the oscillatory flow locally becomes very similar to the static flow and Eq. (3) becomes (here and in what follows the term $\mathbf{r}$ is omitted)

$$
\phi\langle\mathbf{v}\rangle_{i}=-\frac{1}{\eta} k_{0 i j} \nabla_{j}\langle p\rangle .
$$

This last relation is the Darcy law. A phenomenological version of this law corresponds to a work by Darcy, ${ }^{16}$ and the components $k_{0 i j}$ define the static viscous permeability tensor.
When $\omega$ becomes very large, the effect of viscosity becomes negligible and $\alpha_{i j}(\omega)$ tends to the tortuosity tensor $\alpha_{\infty i j}$. $\alpha_{i j}(\omega)$ and $k_{i j}(\omega)$ have been shown to be symmetric. ${ }^{17}$

\section{B. Summary of numerical methods}

In order to describe the periodic oscillating flow created in a porous medium by an external unit harmonic pressure gradient $\mathbf{e} e^{-i \omega t}$, one has to solve the following set of scaled equations:

$$
\begin{aligned}
& \frac{-i \omega}{\nu} \mathbf{w}=-\nabla \pi+\Delta \mathbf{w}+\mathbf{e} \quad \text { in } \Omega_{f}, \\
& \nabla \cdot \mathbf{w}=0 \quad \text { in } \Omega_{f}, \\
& \mathbf{w}=0 \quad \text { on } \partial \Omega,
\end{aligned}
$$

where $\mathbf{e}$ is a unit vector and $\nu=\eta / \rho_{0}$. The solution to the problems (5)-(7) is fixed by adding the condition that $\pi$ is a spatially stationary or periodic field. This unsteady Stokes problem is relevant to sound propagation as long as the wavelength is large enough for the saturating fluid to behave as an incompressible fluid in volumes of the order of the homogenization volume (a period in the case of periodic structure).

Writing the pressure $p$ in terms of its mean and deviatoric parts, $p=\langle p\rangle+\Pi,\langle\Pi\rangle=0$, the macroscopic pressure gradient in Eqs. (2) and (3) is related to e in Eq. (5) by

$$
\nabla\langle p\rangle=-|\nabla\langle p\rangle| \mathbf{e}
$$

the small fluctuation $\Pi$ is related to $\pi$ in Eq. (2) by

$$
\Pi(\mathbf{x})=|\nabla\langle p\rangle| \pi(\mathbf{x}),
$$

and $\mathbf{v}$ is related to $\mathbf{w}$ by

$$
\mathbf{v}=|\nabla\langle p\rangle / \eta| \mathbf{w}(\mathbf{x}) \text {. }
$$

From Eqs. (8)-(10) and (3), the viscous permeability components are given by

$$
\phi\left\langle\mathbf{w}_{i}\right\rangle=k_{i j}(\omega) e_{j} .
$$

By using three individual solicitation vectors $\mathbf{e}^{i}$ in three perpendicular directions, with components $\mathbf{e}_{j}^{i}=\delta_{i j}$, Eq. (11) may be rewritten as

$$
k_{i j}(\omega)=\phi\left\langle\mathbf{w}_{i}^{j}\right\rangle
$$

where $\mathbf{w}_{i}^{j}$ is solution to

$$
\frac{-i \omega}{\nu} \mathbf{w}_{i}^{j}=-\nabla_{i} \pi^{j}+\Delta \mathbf{w}_{i}^{j}+\delta_{i j} \quad \text { in } \Omega_{f},
$$

with $\pi^{j}$ stationary or periodic and $\mathbf{w}^{j}$ verifying the conditions (6) and (7). Equation (12) can also take the following forms:

$$
k_{i j}(\omega)=\phi\left\langle\mathbf{w}_{l}^{j} \delta_{i l}\right\rangle=\phi\left\langle\mathbf{w}_{l}^{j} \mathbf{e}_{l}^{i}\right\rangle=\phi\left\langle\mathbf{w}^{j} \cdot \mathbf{e}^{i}\right\rangle .
$$

At $\omega=0$, Eqs. (5) $-(7)$, with the condition that $\pi$ is a stationary field, simply describe the viscous fluid motion in steady state regime. This is the steady Stokes problem for periodic structures, where $\mathbf{w}_{0}$ is the scaled static velocity field in the pore in $\mathrm{m}^{2}$. Thus, it derives from Eq. (14) that the 
components $k_{0 i j}$ defining the static viscous permeability in three perpendicular directions, $\mathbf{e}_{j}^{i}=\delta_{i j}$, are simply given by

$$
k_{0 i j}=\phi\left\langle\mathbf{w}_{0}^{j} \cdot \mathbf{e}^{i}\right\rangle .
$$

At the opposite frequency range, when $\omega$ becomes very large, the viscous boundary layer becomes negligible and the fluid tends to behave as a perfect one, having no viscosity. In these conditions, the perfect incompressible fluid formally behaves according to the electric problem. ${ }^{8}$ The components $\alpha_{\infty i j}$ defining the tortuosity in the same perpendicular directions, $\mathbf{e}_{j}^{i}=\delta_{i j}$, are now given by ${ }^{15}$

$$
\alpha_{\infty i j}^{-1}=\left\langle\mathbf{E}^{j} \cdot \mathbf{e}^{i}\right\rangle,
$$

where $\alpha_{\infty i j}^{-1}$ is the inverse of the tortuosity tensor $\alpha_{\infty i j}$, and $\mathbf{E}$ is the scaled electric field that solves the corresponding electrical conduction problem for a porous medium filled with a conducting fluid and having an insulating solid phase, i.e.,

$$
\begin{aligned}
& \mathbf{E}=-\nabla \varphi+\mathbf{e} \quad \text { in } \Omega_{f}, \\
& \nabla \cdot \mathbf{E}=0 \quad \text { in } \Omega_{f}, \\
& \mathbf{E} \cdot \mathbf{n}=0 \quad \text { on } \partial \Omega,
\end{aligned}
$$

and $\varphi$ is a spatially stationary or periodic scalar field representing the deviatoric part of the electric potential.

In the case of periodic porous structures, permeability and tortuosity tensors reduce to a scalar and only one dynamic quantity is finally necessary to represent the viscous dissipation properties of a given material. ${ }^{17}$ An example is given in what follows where the dynamic viscous permeability is computed for a 2D periodic foam model geometry, which conserves the purely geometrical macroscopic parameters found with a 3D $\mu \mathrm{CT}$ analysis.

\section{APPLICATION TO AN OPEN-CELL ALUMINUM FOAM}

\section{A. Basic inverse 2D foam model geometry}

One approach commonly used to relate the local geometry parameters of the porous material and its physical behavior involves the development of microstructural models. This implies the assumption of unit cell geometries. ${ }^{18}$ Furthermore, if the microstructure results from a known mechanism, it is appealing to directly incorporate this knowledge in the simulation procedure. ${ }^{19}$ For instance, foams generally result from the nucleation, growth, and expansion of gas bubbles in a melt or reacting liquid system. ${ }^{20}$ Scientists and mathematicians have been contemplating the structure of soap froth for over a century, often focusing on idealized systems that are ordered and monodisperse in response to the Kelvin $^{21}$ problem: partitioning 3D space into equal-volume cells and minimum surface area. ${ }^{22}$ Real foams, by contrast, are disordered, and contain an impressive variety of cell shapes, even when the bubbles have equal volume. ${ }^{23}$ However, the complications encountered in trying to identify suitable unit cells that are representative of complex macrostructures of real foams generally lead to consider simple geometries for these unit cells. ${ }^{24}$ Zheng and Ashby ${ }^{25}$ argued that the cell geometry best representing isotropic foams, and

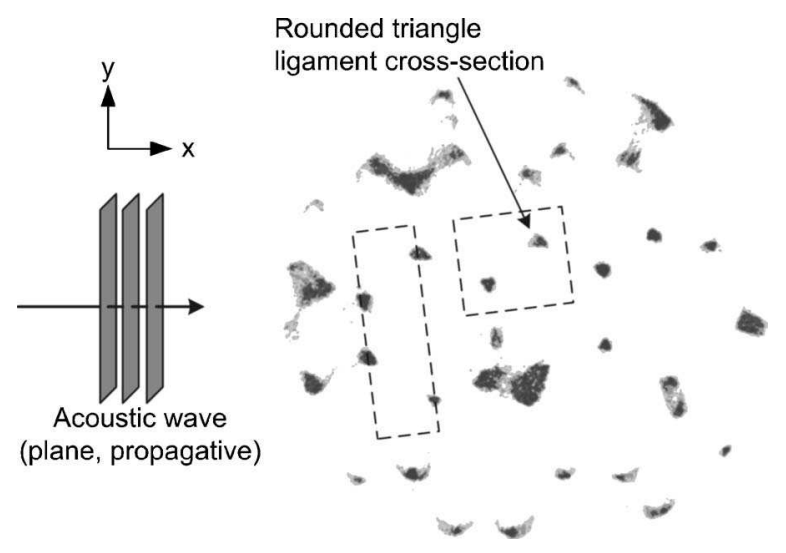

FIG. 1. Virtual slice of a $10 \mathrm{~mm}$ in diameter 40 ppi aluminum foam sample obtained by x-ray axial microtomography (Ref. 1).

at the same time capable of filling space, is a tetrakaidecahedron, also known as the Kelvin cell. ${ }^{26}$ This truncated octahedron was considered the best unit cell for partitioning space into cavities of equal volumes while minimizing the interfacial area until 1993, where Weaire and Phelan discovered a foam structure containing two different types of cavities of equal volumes and with a smaller surface area than the Kelvin foam. ${ }^{27}$ But whether it is the best monodisperse foam still remains an open question.

In this study, it is intended to find a simple equivalent 2D periodic foam model geometries from 3D microstructural information provided by $\mu \mathrm{CT}$ analysis. It is thus somewhat straightforward to consider the honeycomb structure as a starting 2D idealized PUC, the two-dimensional counterpart of the Kelvin cell, which has been recently proved to be the best partition for paving the two-dimensional space into equal-surface cells and minimum length perimeter. ${ }^{28}$ Furthermore, this argument is supported by Fig. 1 showing a cross section of the studied $92 \%$ porosity 40 ppi Duocel open-cell aluminum foam, where ligaments are clearly organized in a hexagonal pattern. In addition, there is experimental evidence that the cross-section shape of a foam ligament is evolving from a circle $(\phi=85 \%)$ for low porosity foams to convex $(\phi \approx 90 \%)$, straight $(\phi=94 \%)$, and concave $(\phi$ $=98 \%$ ) triangles for high porosity foams. ${ }^{29}$

Following the previous discussion, the simplest model one can find for the studied aluminum foam may be obtained by assuming ligaments of circular cross section arranged in the hexagonal pattern as shown in Fig. 2. In this particular case, the characteristic dimensions $l=2 \Lambda^{\prime} \sqrt{\pi(1-\phi) / 3 \sqrt{3}} / \phi$ $\approx 884 \mu \mathrm{m}$ and $r=(1-\phi) \Lambda^{\prime} / \phi \approx 161 \mu \mathrm{m}$ of the hexagonal porous geometry were found by inversion from the knowledge of $\phi=0.92$ and $\Lambda^{\prime} \approx 1.85 \mathrm{~mm}$ deduced by 3D $\mu \mathrm{CT}$ analysis; ${ }^{1}$ here $\Lambda^{\prime}$ is the thermal characteristic length defined as the fluid phase volume to wet surface ratio. In generating this inverse basic model, the aim was to create the simplest 2D foam model geometry which conserves the purely geometrical macroscopic parameters deduced from 3D microstructural analysis.

\section{B. Numerical computations}

Numerical computations were performed on the smallest periodic rectangles $R_{H}$ and $R_{V}$ of the 2D periodic foam 


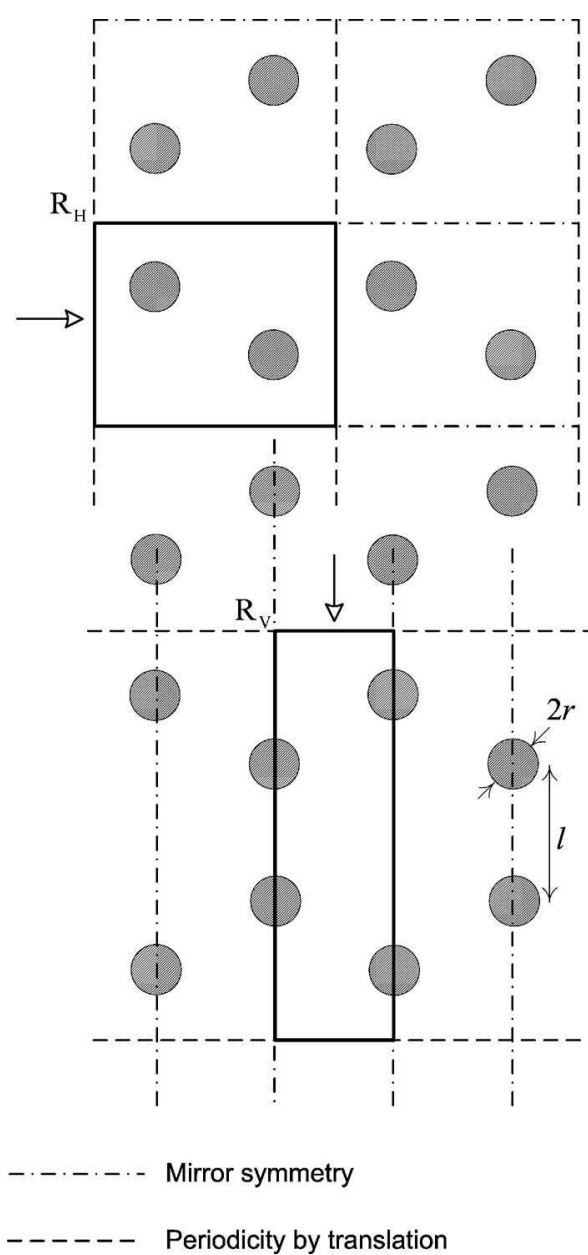

FIG. 2. Basic 2D periodic foam model geometry.

model geometry depicted in Fig. 2. Using a finite element commercial code ${ }^{30}$ macroscopic parameters $\phi, \Lambda^{\prime}, \Lambda, k_{0}$, $\alpha_{\infty}$, and $\alpha_{0}$ were computed. The results are listed in Table I. The open porosity $\phi$ was computed from the volume of the mesh, and the thermal characteristic length $\Lambda^{\prime}$ was obtained by the volume to wet surface ratio of the mesh. The static viscous permeability $k_{0}$ and the static viscous tortuosity $\alpha_{0}$ were computed from the steady Stokes problem using Eq. (15), and by means of

$$
\alpha_{0}=\left\langle\mathbf{w}_{0}^{2}\right\rangle /\left\langle\mathbf{w}_{0}\right\rangle^{2} .
$$

An equivalent result has been obtained by Norris ${ }^{31}$ on the basis of homogenization theory, and it has been formally

TABLE I. Comparison between computed and measured macroscopic parameters.

\begin{tabular}{lcc}
\hline \hline \multicolumn{1}{c}{ Macroscopic parameters } & Computations & Measurements \\
\hline$\phi(-)$ & 0.92 & $0.91 \pm 0.01^{\mathrm{a}}$ \\
$\Lambda^{\prime}(\mathrm{mm})$ & 1.85 & $2.01 \pm 0.43^{\mathrm{b}}$ \\
$\Lambda(\mathrm{mm})$ & 1.00 & $0.99 \pm 0.06^{\mathrm{b}}$ \\
$k_{0}\left(\times 10^{-8} \mathrm{~m}^{2}\right)$ & 4.83 & $10.39 \pm 1.23^{\mathrm{c}}$ \\
$\alpha_{\infty}(-)$ & 1.08 & $1.07 \pm 0.01^{\mathrm{b}}$ \\
$\alpha_{0}(-)$ & 1.30 & $\cdots$ \\
\hline
\end{tabular}

${ }^{\mathrm{a}}$ Reference 36.

${ }^{\mathrm{b}}$ Reference 33 .

${ }^{\mathrm{c}}$ Reference 37. expressed by Lafarge in this way by using ideas borrowed from macroscopic electrodynamics. ${ }^{15,32}$ No-slip boundary conditions at the pore walls and periodicity of $\mathbf{w}_{0}$ and $\pi_{0}$ were prescribed. Additional Neumann boundary conditions are set in the remaining lateral borders due to the symmetries of the problems. The high frequency viscous tortuosity $\alpha_{\infty}$ and the viscous characteristic length $\Lambda$ were computed using Eq. (16) and the definition of Johnson et al., ${ }^{6}$

$$
2 / \Lambda=\int_{\partial \Omega} \mathbf{E}^{2} d S / \int_{\Omega} \mathbf{E}^{2} d V
$$

which introduced this length-scale parameter $\Lambda$ as the weighted pore volume $\left(\Omega_{p}\right)$ to pore surface $\left(\Omega_{s}\right)$ ratio. Neumann boundary conditions on the fluid-solid interface and periodicity on the inlet-outlet surfaces were used for $\varphi$. Neumann boundary conditions are set in the remaining lateral borders. The symmetry property of the viscous permeability tensor ${ }^{17}$ is used to evaluate the uncertainty on macroscopic parameters. The relative differences found for the horizontal and vertical directions are less than $0.07 \%$ and the nondiagonal terms are actually numerically equal to zero.

The full unsteady Stokes problem was solved using the same finite element code. No-slip boundary conditions at the pore walls and periodicity of $\mathbf{w}(\omega)$ and $\pi$ were prescribed. The number of elements and their distribution in the fluid phase regions of $R_{H}$ and $R_{V}$ were varied, with attention paid especially to the throat and the near-wall areas, to examine the accuracy and convergence of the scaled velocity field solutions. A total of $5 \times 10^{4}$ elements were used to guarantee the variation of the solutions to within a fraction of a percent when the distribution and number of elements were varied. Figure 3 gives an idea of the mesh used at the highest value of the frequency. The mesh corresponding to the PUC domain is plotted together with a closeup of the interface. The present work considered quadratic Lagrange elements. Once the scaled velocity fields are known, the dynamic viscous permeability is computed according to Eq. (14). Results are presented in Fig. 4, where $k(\omega)$ is plotted. Once again, the symmetry property of the viscous permeability tensor ${ }^{17}$ is used to evaluate the uncertainty on $k(\omega)$. The relative differences found for the horizontal and vertical directions are less than $5 \times 10^{-6}$ and the nondiagonal terms are also numerically equal to zero. In Fig. 4, the frequency dependence of the calculated $k(\omega)$ is also compared to the generic asymptotic dependences given by the models of Johnson et al. ${ }^{6}$ (JKD) and Pride et al. ${ }^{11}$ (PMG). The authors ${ }^{6,11}$ suggest the following expressions for the dynamic viscous permeability:

$$
k(\omega)=\frac{i \eta \phi}{\alpha(\omega) \omega \rho_{0}},
$$

and

$$
\alpha(\omega)=\alpha_{\infty}\left[1+\frac{1}{i \varpi} f(\varpi)\right],
$$

where $\varpi$ is a dimensionless viscous angular frequency given by 

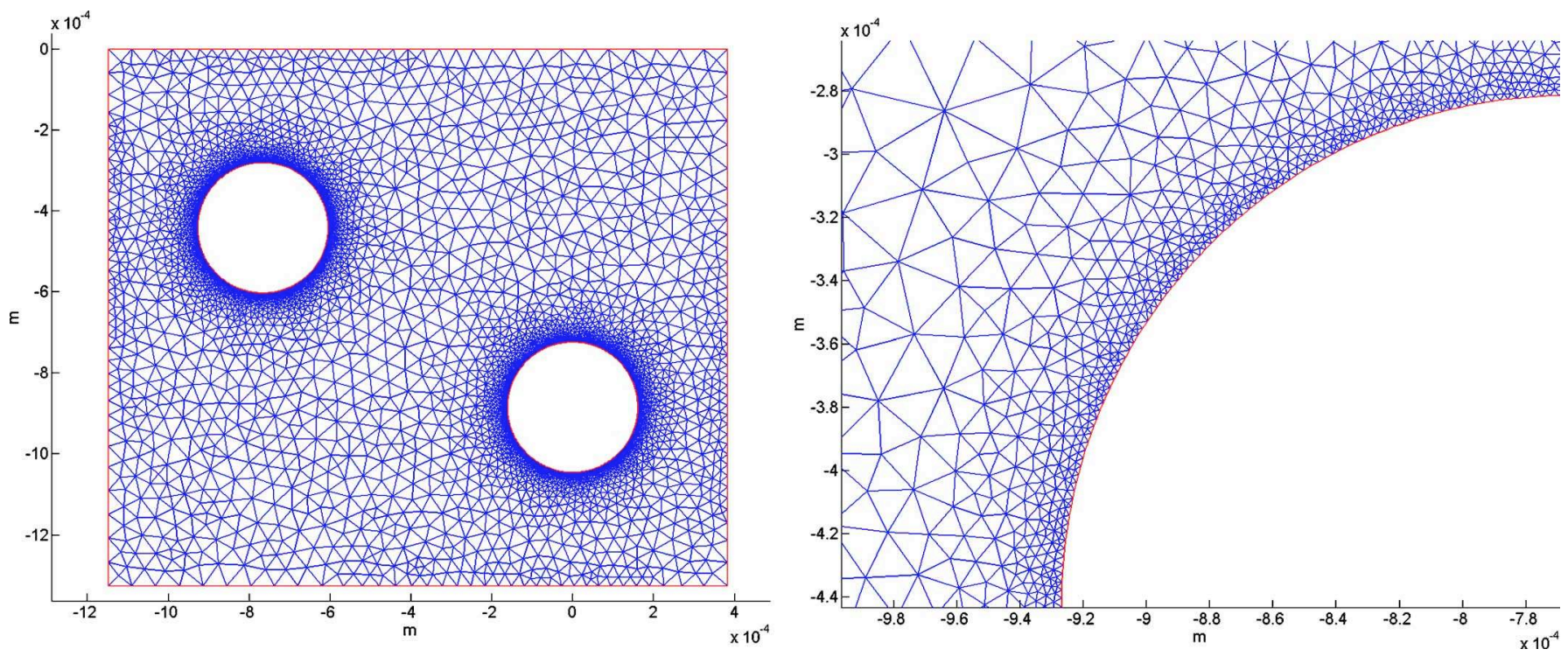

FIG. 3. (Color online) PUC meshed domain (left) and closeup of the interface (right).

$$
\varpi=\frac{\omega}{\nu} \frac{k_{0} \alpha_{\infty}}{\phi},
$$

and with the following shape function:

$$
f(\varpi)=1-P+P \sqrt{1+\frac{M}{2 P^{2}} i \varpi,}
$$

where the dimensionless shape factors have been introduced,

$$
\begin{aligned}
& M=\frac{8 k_{0} \alpha_{\infty}}{\Lambda^{2} \phi}, \\
& P=\frac{M}{4\left(\left(\alpha_{0} / \alpha_{\infty}\right)-1\right)} .
\end{aligned}
$$

It can be seen that for $P=1$, the PMG model reduces to the JKD model. As expected, PMG model is correcting JKD model to obtain the proper low frequency asymptotic behavior of the calculated $k(\omega)$ imaginary part by the introduction of $\alpha_{0}$. As an illustration, we show, respectively, in Fig. 5 the horizontal components of the steady Stokes and unsteady Stokes at $400 \mathrm{~Hz}$, and the electric scaled patterns obtained for excitation along the horizontal direction of the periodic geometry. As previously noticed by several authors, such as Martys and Garboczi, ${ }^{9}$ due to the nonslip condition, the fluid flow paths are clearly more concentrated that do the electriccurrent paths arising when effects of fluid viscosity are minimal. As frequency increases, the scaled patterns tend to be more and more homogeneous with decreasing stagnant areas.

\section{Experimental results}

We report here experimental measurements of dynamic tortuosity and macroscopic parameters, performed on the studied Duocel 40 ppi open-cell aluminum foam sample. The measurement of $\alpha_{\infty}, \Lambda$, and $\Lambda^{\prime}$ is based on an inversion technique. ${ }^{33}$ The inversion principle consists in minimizing differences between measured and estimated impedances. Measured impedance is based on a standard test method described in ASTM E1050-86 for impedance and absorption of

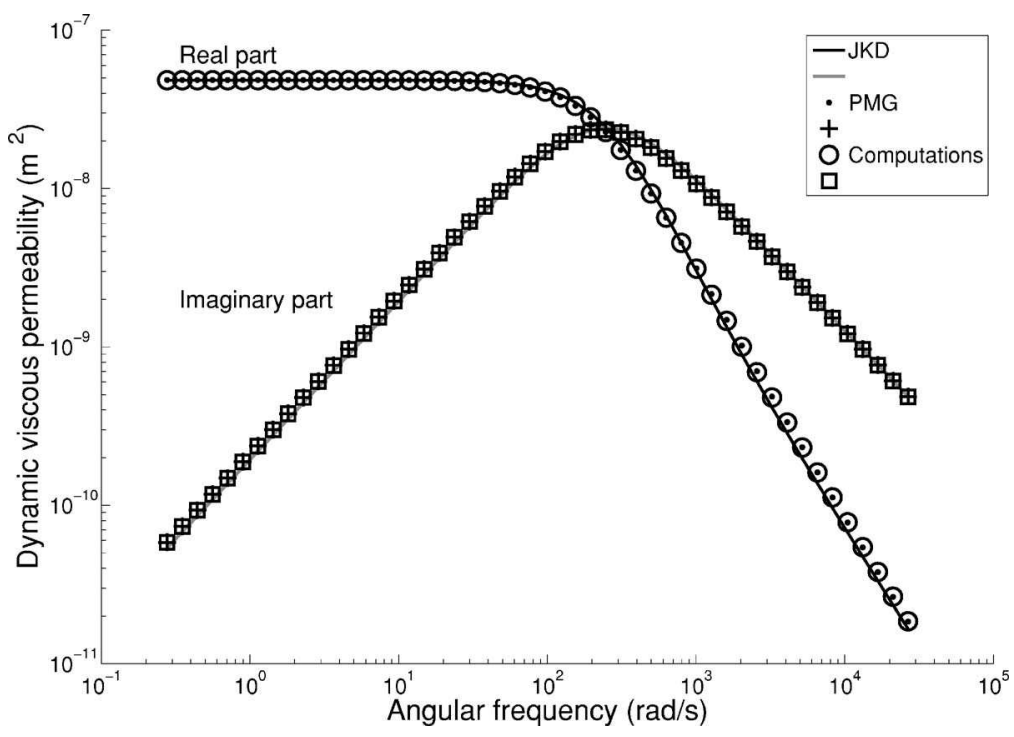

FIG. 4. Dynamic viscous permeability of the basic 2D periodic foam model geometry: numerical results and comparison with the models of Johnson et al. (JKD) (Ref. 6) and Pride et al. (PMG) (Ref. 11). 
Steady Stokes scaled velocity field

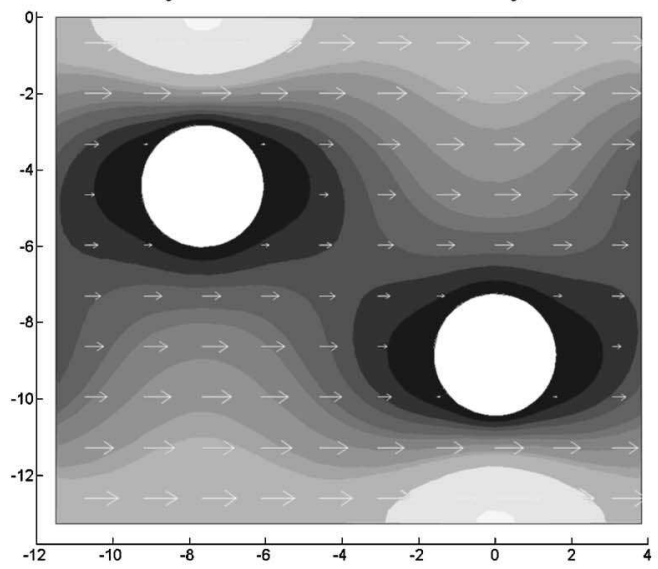

Unsteady Stokes scaled velocity field

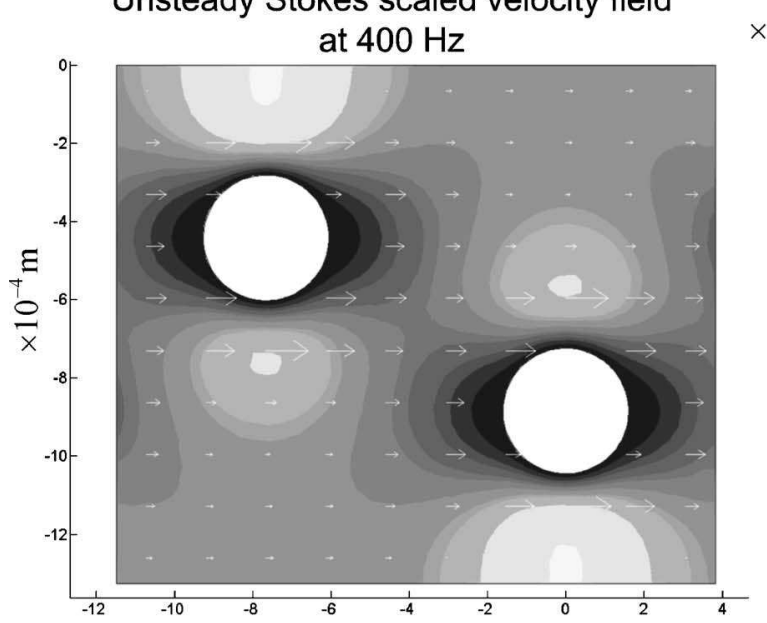

Scaled electric field

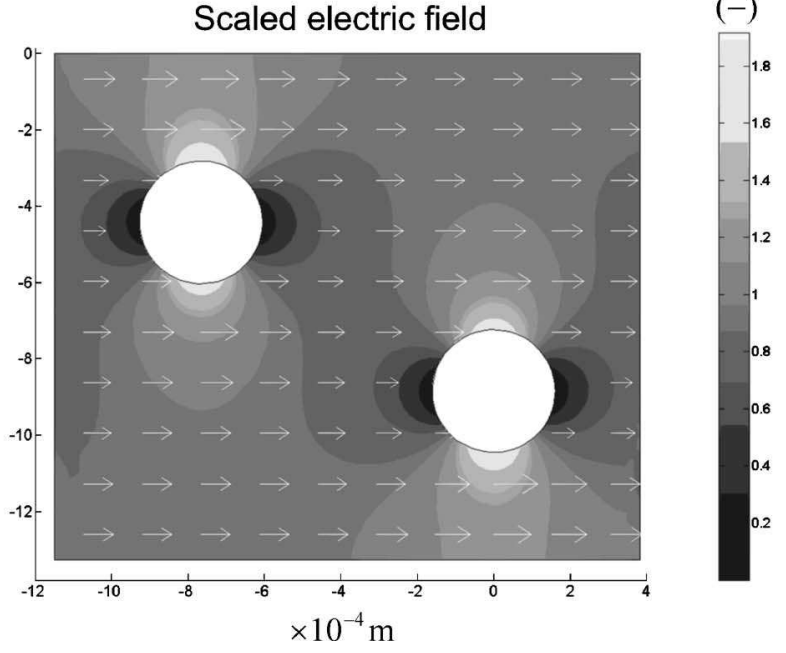

FIG. 5. Scaled fields evolution with frequency.

acoustical materials using a tube, two microphones, and a digital frequency analysis system. Estimated impedance is deduced from dynamic viscous and thermal dissipation properties taken into account through the models of Johnson et $a .^{6}{ }^{6}$ and Champoux and Allard, ${ }^{34}$ respectively. Estimated impedance is derived from the least mean square fit of a function of the triplet $\left(\alpha_{\infty}, \Lambda, \Lambda\right.$, $)$ on the frequency range of interest, while satisfying physical restrictions on these parameters. Furthermore, it is worth noting that, considering the large cell size of the studied sample, transition frequencies of the medium are very low, on the order of a few tenths of hertz. This means that, for this specific material, the inversion has been realized on the high frequency regime of the porous medium. As a consequence, the thermal characteristic length $\Lambda^{\prime}$ determined by this inversion technique is the same as the one which would have been found if Lafarge ${ }^{35}$ model had been implemented.

Measured macroscopic parameters are also listed in Table I. The porosity $\phi$ is measured according to the so called missing mass method ${ }^{36}$ based on mass measurement differences of the porous bulk in air and vacuum, using a vacuum air pump allowing to pump out air from a tank down to an absolute pressure of $0.2 \mathrm{psi}$ and a balance of readability of $0.001 \mathrm{~g}$. The experimental value of the static permeability $k_{0}$ is obtained by means of accurate measurements of differential pressures across serial mounted calibrated and unknown flow resistances, with a controlled steady and nonpulsating laminar volumetric air flow as described by Stinson and Daigle. ${ }^{37}$ Measured macroscopic parameter values are comparable to those obtained with numerical computations performed on the basic 2D periodic foam model geometry, except for the static permeability $k_{0}$ (resistivity $\sigma=\eta / k_{0}$ ) which is underestimated (overestimated) by the 2D foam model by a factor of 2 . This can be explained by the dimensionality of the model. In our 2D model, ligaments are always perpendicular to the flow direction, whereas in a threedimensional space, ligaments of the real open-cell foam sample also adopt other spatial orientations, that is, increasing (decreasing) the permeability (resistivity) of the real medium. This result is in accordance with other literature data reported for fibrous media made from circular cross-section fibers having porosities greater than $90 \%$. For a flow parallel to the fiber direction, permeability $k_{0 \mid}$ is approximately equal to twice the reported values $k_{0} \perp$ for a flow perpendicular to the fiber direction, $k_{0 \|} \approx 2 \times k_{0} \perp$ (see, for example, Refs. 38 and 39). This property can eventually be used to correct the found prediction with a 2D model. In our case, because of the large cell size, the order of magnitude of the measured resistivity is so low $\left(\approx 177 \mathrm{~N} \mathrm{~m}^{-4} \mathrm{~s}\right)$ that an error made on its estimation, with a factor approximately equal to two $\left(\approx 381 \mathrm{~N} \mathrm{~m}^{-4} \mathrm{~s}\right)$, will have small influence on the resulting dynamic dissipation properties such as $k(\omega)$ or $\alpha(\omega)$.

Next, the dynamic viscous permeability of the 2D periodic foam model geometry is determined. From the previously computed macroscopic parameters, Fig. 6 shows the JKD and PMG dynamic permeabilities $k(\omega)$ compared to impedance tube measurements. The dynamic permeability measurements were obtained using a $44.4 \mathrm{~mm}$ impedance tube with the two-cavity technique, ${ }^{40}$ where the frequency dependant surface impedance and propagation constant (from which all the pertaining dynamic macroscopic quantities can be obtained-see, for example, Lafarge et al. ${ }^{35}$ for the detailed relationships) of the material are calculated from a set of distinct acoustic impedances derived by measurements taken at the surface of the porous material and achieved by simply changing the air space depth behind the porous material. There is good agreement between measured and modeled dynamic permeabilities, especially at higher 


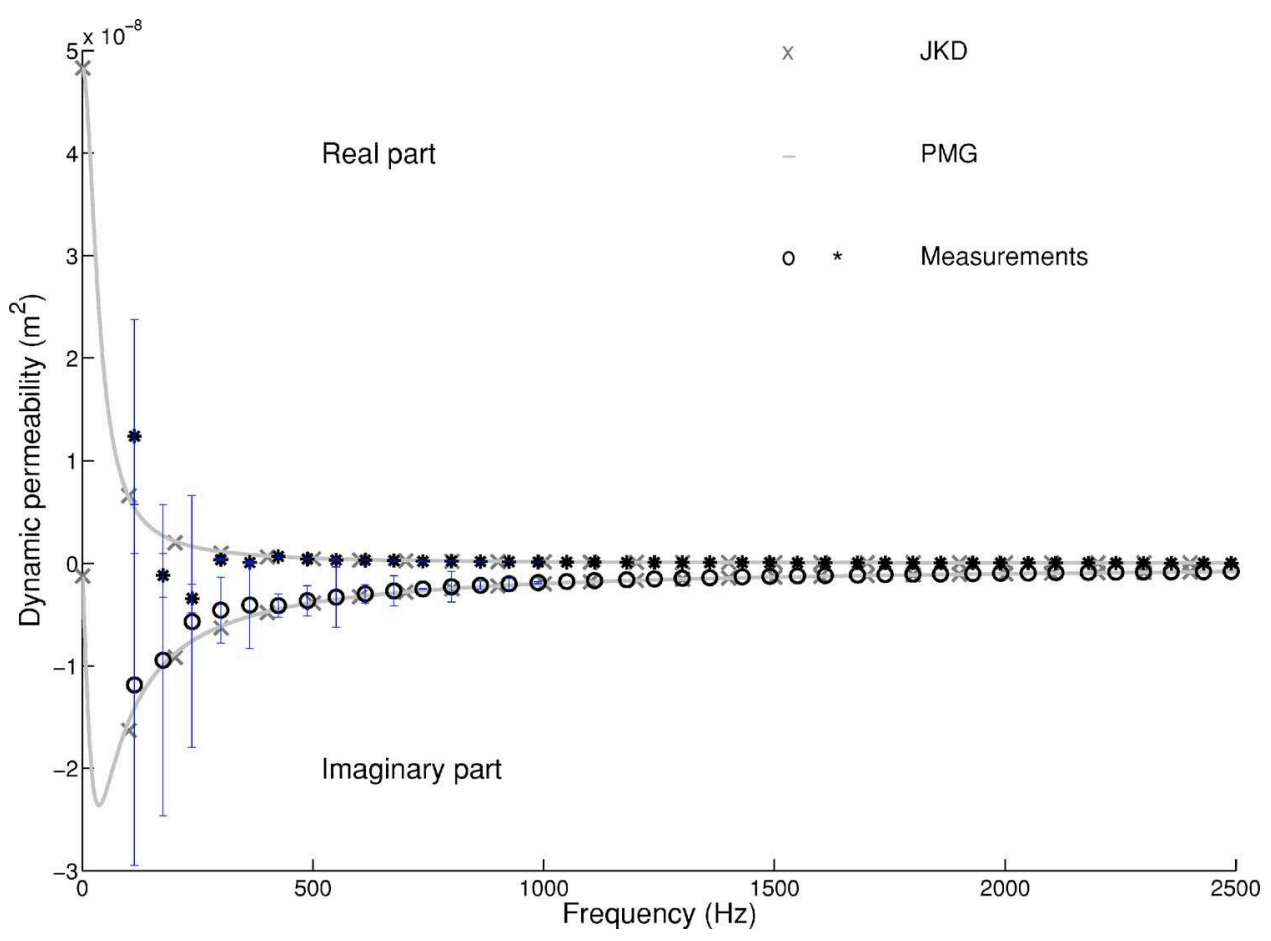

FIG. 6. (Color online) Dynamic permeability: comparison between measurements performed on a real opencell aluminum foam sample and microstructural predictions based on an idealized 2D foam geometry with the models of Johnson et al. (JKD) (Ref. 6) and Pride et al. (PMG) (Ref. 11). Error bars represent the standard deviations of the measurements under $1000 \mathrm{~Hz}$ for different real samples of the same foam. The increasing uncertainty is due to the decreasing sensitivity on the phase measurement for longer wavelengths. frequencies. One can note that the numerical computations predict a transition frequency around $40 \mathrm{~Hz}$. This very low transition frequency is due to large cell size of the foam. Using the experimental setup, it was not possible to measure the low frequency behavior (need to increase significantly the intermicrophonic distance in order to keep the proper sensitivity on the wavelength around the transition and in the low frequency regimes); however, the experimental results in the measurable frequency range $(400-2500 \mathrm{~Hz})$ are in good accordance with the predicted asymptotic inertial regime.

\section{DISCUSSION AND SUMMARY}

The utility of using 2D periodic model geometry to predict the dynamic viscous dissipation properties of real porous materials is unquestionable, as numerical simulations are usually easier and faster to make, as long as microstructural information or purely geometric macroscopic parameters can be readily determined on the real samples. For open-cell foams, the microstructural information is analyzed to generate a three-dimensional periodic unit cell, so that purely geometrical macroscopic parameters $\left(\phi, \Lambda^{\prime}\right)$ are determined, and a basic periodic foam model geometry built by inversion.

The question that this paper has focused on is the limit of relating dynamic viscous dissipation properties of porous media and simplified 2D periodic model geometry. The principal contribution of the present work is that all relevant quantities have been computed on a basic periodic foam model geometry, from the basis of a real open-cell aluminum foam sample analyzed by $\mathrm{x}$-ray microtomography. The obtained results tend to demonstrate the important effect that the dimensionality of the geometrical model has on the static viscous permeability of the porous structure. The $2 \mathrm{D}$ periodic foam model geometry considered in this paper forces the foam ligaments to be perpendicular to the flow direction, so that the permeability is artificially decreased. However, in real foam structures, with various ligament orientations, the ligaments will tend to adopt various spatial orientations, less resistive than if they were all perpendicular to the flow direction.

In summary, for a $2 \mathrm{D}$ periodic open-cell foam model geometry, the dynamic viscous permeability $k(\omega)$ and four asymptotic macroscopic parameters $\left(k_{0}, \alpha_{0}, \Lambda, \alpha_{\infty}\right)$ were computed. It was shown that a relatively good estimate of these quantities can be obtained from a basic 2D geometrical model, except in the low frequency range, where the low dimensionality of the model is leading to overestimated values of the permeability. However, the knowledge of this property could be used to correct this behavior in order to give reasonably good predictions for the dynamic viscous dissipation properties of an open-cell foam. More generally, this result tends to promote the idea of a three-dimensional implementation for the computation of the dynamic viscous dissipation properties. Nevertheless, the presented experiments have been done for a relatively narrow range of variation of the dynamic tortuosity. Future experiments will include the investigation of smaller cell sizes porous media on the order of $\sim 0.1 \mathrm{~mm}$ for which the transition frequencies will be easily measurable with a classical impedance tube or the use of a long tube [a rolled pipe of more than $50 \mathrm{~m}$ (Ref. 41)]. This method can also be used as a bottom-up approach for microstructure optimization of $2 \mathrm{D}$ sound absorbing materials.

\section{ACKNOWLEDGMENTS}

This work was supported in part by grants-in-aid from Alcan, CQRDA, NSERC, Auto21, and Région Rhône-Alpes. A part of the research presented in this paper was also financed by the Fonds Québécois de la Recherche sur la Nature et les Technologies (FQRNT) by the intermediary of the 
Aluminium Research Centre-REGAL. Camille Perrot would like to thank Igor Jovet for his helpful contributions in the development of the numerical programs.

${ }^{1}$ C. Perrot, R. Panneton, and X. Olny, J. Appl. Phys. 101, 113538 (2007).

${ }^{2}$ C. Perrot, R. Panneton, and X. Olny, J. Appl. Phys. 102, 074917 (2007)

${ }^{3}$ L. D. Landau and E. M. Lifshitz, Fluid Dynamics (Pergamon, Oxford, 1959), p. 95.

${ }^{4}$ D. A. P. Jayasinghe, S. M. Letelier, and H. J. Leutheusser, Int. J. Mech. Sci. 16, 819 (1974).

${ }^{5}$ P. Sheng and M.-Y. Zhou, Phys. Rev. Lett. 61, 1591 (1988).

${ }^{6}$ D. L. Johnson, J. Koplik, and R. Dashen, J. Fluid Mech. 176, 379 (1987).

${ }^{7}$ E. Charlaix, A. P. Kushnick, and J. P. Stokes, Phys. Rev. Lett. 61, 1595 (1988).

${ }^{8}$ R. J. S. Brown, Geophysics 45, 1269 (1980).

${ }^{9}$ N. Martys and E. J. Garboczi, Phys. Rev. B 46, 6080 (1992).

${ }^{10}$ A. M. Chapman and J. J. L. Higdon, Phys. Fluids A 4, 2099 (1992).

${ }^{11}$ S. R. Pride, F. D. Morgan, and A. F. Gangi, Phys. Rev. B 47, 4964 (1993).

${ }^{12}$ M. Firdaouss, J.-L. Guermond, and D. Lafarge, Int. J. Eng. Sci. 36, 1035 (1998).

${ }^{13}$ A. Cortis, D. M. J. Smeulders, J. L. Guermond, and D. Lafarge, Phys. Fluids 15, 1766 (2003).

${ }^{14}$ S. Gasser, F. Paun, and Y. Brechet, J. Acoust. Soc. Am. 117, 2090 (2005).

${ }^{15}$ D. Lafarge, in Matériaux et Acoustique 1, edited by M. Bruneau and C. Potel (Lavoisier, Paris, 2006).

${ }^{16} \mathrm{H}$. Darcy, Les Fontaines Publiques de la Ville de Dijon (Victor Dalmont, Paris, 1856).

${ }^{17}$ J.-L. Auriault, L. Born, and R. Chambon, J. Acoust. Soc. Am. 77, 1641 (1985).

${ }^{18}$ S. Mullens, J. Luyten, and J. Zeschky, in Cellular Ceramics, edited by M. Scheffler and P. Colomo (Wiley, Weinheim, 2005).

${ }^{19}$ J.-F. Thovert, F. Yousefian, P. Spanne, C. G. Jacquin, and P. M. Adler, Phys. Rev. E 63, 061307 (2001).

${ }^{20}$ A. Cunningham and N. C. Hilyard, in Low Density Cellular Plastics, edited by N. C. Hilyard and A. Cunningham (Chapman and Hall, Cambridge, 1994).

${ }^{21}$ D. W. Thompson, in On Growth and Form, abridged ed., edited by J. T. Bonner, (Cambridge University Press, Cambridge, 1961).

${ }^{22}$ A. M. Kraynik, D. A. Reinelt, and F. van Swol, Phys. Rev. Lett. 93, 208301 (2004).

${ }^{23}$ E. B. Matzke, Am. J. Bot. 33, 58 (1946).

${ }^{24}$ L. J. Gibson and M. F. Ashby, Cellular Solids: Structure and Properties (Cambridge University Press, Cambridge, 1988).

${ }^{25} \mathrm{~J}$. Zheng and M. F. Ashby, "Theoretical studies on isotropic foams," Cambridge University Engineering Department Report No. CUED/C-Mats/Tr 158, 1989, Cambridge University, Cambridge, U.K..

${ }^{26}$ Lord Kelvin (Sir William Thompson), Philos. Mag. 24, 503 (1887).

${ }^{27}$ D. Weaire and R. Phelan, Philos. Mag. Lett. 69, 107 (1994).

${ }^{28}$ T. C. Hales, Discrete Comput. Geom. 25, 1 (2001).

${ }^{29}$ A. Bhattacharya, V. V. Calmidi, and R. L. Mahajan, Int. J. Heat Mass Transfer 45, 1017 (2002).

${ }^{30}$ COMSOL 3.3, WTC-5 pl. Robert Schuman, 38000 Grenoble France.

${ }^{31}$ A. N. Norris, J. Wave-Mater. Interact. 1, 365 (1986).

${ }^{32} \mathrm{D}$. Lafarge, "Propagation du son dans les matériaux poreux à structure rigide saturéspar un fluide viscothermique," $\mathrm{Ph}$. D. Thesis, Université du Maine, 1993.

${ }^{33}$ Y. Atalla and R. Panneton, Can. Acoust. 33, 11 (2005).

${ }^{34}$ Y. Champoux and J. F. Allard, J. Appl. Phys. 70, 1975 (1991).

${ }^{35}$ D. Lafarge, P. Lemarinier, J. F. Allard, and V. Tarnow, J. Acoust. Soc. Am. 102, 1995 (1997).

${ }^{36}$ R. Panneton and E. Gros, Acta. Acust. Acust. 91, 342 (2005).

${ }^{37}$ M. R. Stinson and G. A. Daigle, J. Acoust. Soc. Am. 83, 2422 (1988)

${ }^{38}$ V. Tarnow, J. Acoust. Soc. Am. 100, 3706 (1996).

${ }^{39}$ V. Tarnow, J. Acoust. Soc. Am. 111, 2735 (2002).

${ }^{40}$ H. Utsuno, T. Tanaka, T. Fujikawa, and A. F. Seybert, J. Acoust. Soc. Am. 86, 637 (1989).

${ }^{41}$ Z. E. A. Fellah, M. Fellah, N. Sebaa, W. Lauriks, and C. Depollier, J. Acoust. Soc. Am. 119, 1926 (2006). 